Research Article

\title{
Study on Pounding Response of Adjacent Inelastic SDOF Structures Based on Dimensional Analysis
}

\author{
Xuyong Chen, ${ }^{1}$ Huipeng Guo, ${ }^{1}$ Tao Wang, ${ }^{1}$ and Qiaoyun $W u \mathbb{D}^{1,2}$ \\ ${ }^{1}$ School of Civil Engineering and Architecture, Wuhan Institute of Technology, Wuhan 430073, China \\ ${ }^{2}$ State Key Laboratory of Geomechanics and Geotechnical Engineering, Institute of Rock and Soil Mechanics, \\ Chinese Academy of Sciences, Wuhan 430071, China
}

Correspondence should be addressed to Qiaoyun Wu; wuqiaoyun@wit.edu.cn

Received 1 December 2020; Revised 9 March 2021; Accepted 16 April 2021; Published 28 April 2021

Academic Editor: Nicola Baldo

Copyright (c) 2021 Xuyong Chen et al. This is an open access article distributed under the Creative Commons Attribution License, which permits unrestricted use, distribution, and reproduction in any medium, provided the original work is properly cited.

\begin{abstract}
The dimensional analysis method is applied to study the pounding response of two inelastic single-degree-of-freedom (SDOF) structures under simplified earthquake excitation. The improved Kelvin pounding model is used to simulate the force and deformation of the collider during the contact process. Using bilinear interstory resistance model to simulate the inelastic characteristics of SDOF structures, the expression of dimensionless pounding force and the dimensionless equation of motion during the pounding process are deduced. When dimensionless parameters are used to represent the colliding equation of adjacent inelastic SDOF structures, the variables affecting the pounding response of the adjacent structures are reduced from 14 to 11, which can clearly reflect the rules during the pounding process. The correctness and superiority of the improved Kelvin model are verified by comparing the pounding responses between the improved Kelvin model and Kelvin model. The pounding response of the two inelastic SDOF structures with improved Kelvin model is illustrated in the form of spectra, and the self-similarity of pounding response of the two inelastic SDOF structures is revealed. The effects of structural parameters on the pounding response are analyzed. The results show that the effects of mass ratio, frequency ratio, and initial spacing between the adjacent inelastic SDOF structures on the pounding response of the left-side structure (with smaller mass and stiffness) are closely related to the division of spectral regions. For the right-side structure with larger mass and stiffness, the amplification of pounding on structural response increases with the increase of mass ratio $\Pi_{m}$ and decreases with the increase of frequency ratio $\mu$ and structural spacing $\Pi_{d}$.
\end{abstract}

\section{Introduction}

Earthquake disaster is one of the most important natural disasters that the human society is facing nowadays, and it is characterized by suddenness and destructiveness. In recent years, earthquake disasters not only cause serious damage or even collapse of building structures but also cause a large number of casualties and huge property losses [1-4]. In the Alaska earthquake of 1964 [5], the Mexico earthquake of 1985 [6], the ChiChi earthquake in Taiwan of 1999 [7], the Wenchuan earthquake of 2008 [8], and the earthquakes in Mexico and Iraq of 2017 [9], a large number of adjacent buildings also suffered serious damage due to poundings during the earthquakes.
Wolf and Skrikerud [10], Chau and Wei [11], and Anagnostopoulos [12] have carried out in-depth research on the collision response of adjacent single-degree-of-freedom structures. Jankowski [13] studied the impact response of a single-degree-of-freedom structural system with nonlinear viscoelastic impact model, analyzed the impact effect on the structural response, and proposed that the nonlinear viscoelastic model can simulate the impact process most accurately by comparison with other impact models. Sabegh [14] and others considered the near-field earthquake and farfield earthquake and, respectively, analyzed the collision problem of two linear single-degree-of-freedom structures in two cases, selected Kelvin model to simulate the collision contact process, and studied the influence of spacing on the 
peak collision force. Zhang et al. [15] simplified the adjacent structure into multiple elastic-plastic single-degree-offreedom structures, analyzed them by precise integration method, and studied the influence of contact element parameters on the collision response. However, the impact of adjacent structures under earthquake action is not only related to the characteristics of ground motion but also affected by the characteristics of structures and the characteristics of adjacent structures. That is to say, structural collision is a high-order nonlinear problem affected by many parameters. Structural pounding is a high-order nonlinear problem affected by many parameters. The main difficulty in studying the pounding response of adjacent structures under earthquake is to deal with a large number of parameters [16]. Dimensional analysis is to determine the relationship between physical quantities according to the principle of dimensional homogeneity of physical laws. Using dimensional analysis method [17] to study structural pounding with fewer dimensionless $\Pi$ parameters can not only reduce the number of parameters but also more clearly reflect the rule of adjacent structural pounding response under earthquake excitation [18].

Makris et al. [18, 19] firstly proposed a dimensional method to study the response of structures under earthquake. Based on the dimensional analysis method, many scholars have studied the pounding problem of adjacent SDOF structures. Zhang and Tang [20] used the dimensional analysis method to study the effect of soil-structure interaction on the structure under earthquake ground motion. In the same year, Dimitrakopoulos et al. [21-23] used the dimensional analysis method to study the pounding reaction between multiple SDOF structures. The Buckingham $\Pi$ theorem was used to characterize the pounding of structures with fewer dimensionless parameters, which reduced the number of parameters affecting the pounding response of the structures. Zhai et al. [16] combined dimensional method with contact element method using Kelvin model to simulate the pounding force. The pounding response between a single oscillator and a rigid barrier was studied and the real-time force and deformation of collider structures in contact phase were described. Wu et al. [24] chose more accurate impact model (improved Kelvin model [25]) to study the pounding response between a single oscillator and a rigid barrier, which made up for the shortcomings of Kelvin impact model and factually revealed the rules of structural pounding. Most of the above studies always simplify adjacent structures to be linear SDOF structures; however, the pounding between adjacent structures is inelastic contact process, so the bilinear model is more realistic.

In this paper, the dimensional analysis method and the improved Kelvin model are used to study the pounding response of two inelastic SDOF structures under simplified seismic excitation. Using bilinear interstory resistance model [26] to simulate the inelastic characteristics of the SDOF structures, the expression of dimensionless pounding force and the dimensionless equation of motion in the pounding process are deduced. The calculated results are compared with those obtained by Kelvin impact model. Finally, the effects of structural parameters (mass ratio, frequency ratio, and initial spacing) on the pounding response are analyzed.

\section{Calculation Models and Dimensionless Equation of Motion}

2.1. Impact Analytical Model. In this paper, an improved Kelvin pounding analytical model is adopted to simulate the pounding force produced by two adjacent inelastic SDOF structures. The improved Kelvin analytical model is illustrated in Figure 1. The expression of the pounding force is as follows [25]:

$$
F=\bar{k} \delta(t)+\bar{c} \dot{\delta}(t)
$$

where $\bar{k}$ is the stiffness coefficient of the impact element, $\delta(t)$ is the relative penetration displacement of two objects in pounding, $\dot{\delta}(t)$ is the penetration velocity, and the timevarying expression of the damping coefficient $\bar{c}$ is as follows:

$$
\bar{c}=\bar{\xi} \delta(t),
$$

in which the mathematical expression of the damping constant $\bar{\xi}$ is as follows:

$$
\bar{\xi}=\frac{3 \bar{k}(1-e)}{2 e\left(V_{1}-V_{2}\right)},
$$

where $e$ represents the coefficient of restitution $(e=1 \mathrm{im}$ plies an elastic pounding without energy loss and $e=0$ implies complete plastic pounding). $V_{1}$ and $V_{2}$ are the velocities of two objects at the moment of collision.

\subsection{Equation of Motions of the Two Inelastic SDOF Systems.} The calculation model of the pounding between two adjacent inelastic SDOF structures under earthquake excitation is shown in Figure 2. The mass, stiffness, and damping parameters of the two SDOF structures are $m_{1}$ and $m_{2}, k_{1}$ and $k_{2}$, and $c_{1}$ and $c_{2}$, respectively. The initial spacing between the two SDOF structures is $d$. In order to study the nonlinearity of the structure, the bilinear interstory resistance model [26] is used to simulate the constitutive relationship of the structures.

When the dimensional analysis method is used to study the pounding response of two inelastic SDOF structures under earthquake, only the acceleration amplitude and angular frequency of ground motion are needed. Therefore, a simplified model can be used to simulate the earthquake excitation. At present, there are two models to simplify the simulations of ground motions, which are harmonic and pulse excitation models [27]. In this paper, the ground motion excitation is assumed as the sinusoidal excitation, and the acceleration amplitude of which is $a_{p}$, and circular frequency is $\omega_{p}$.

Under the action of sinusoidal excitation, the equations of motion of two inelastic SDOF structures are as follows: 


$$
\left\{m_{1} \ddot{X}_{1}(t)+c_{1} \dot{X}_{1}(t)+F_{s 1}(t)+F_{p 12}=-m_{1} \ddot{X}_{g}(t), m_{2} \ddot{X}_{2}(t)+c_{2} \dot{X}_{2}(t)+F_{s 2}(t)-F_{p 12}=-m_{2} \ddot{X}_{g}(t) .\right.
$$

where $\ddot{X}_{g}(t)$ is the excitation acceleration, and $\ddot{X}_{g}(t)=a_{p} \sin \left(\omega_{p} t\right) ; X_{1}(t)$ and $X_{2}(t)$ are the displacement responses of the left-side $\left(m_{1}\right)$ and right-side $\left(m_{2}\right)$ structures at different time $t$, respectively. $F_{s 1}(t)$ and $F_{s 2}(t)$ are the inelastic resistance of the left-side and right-side structures at different time $t$, respectively. The incremental form of the inelastic resistance is $\Delta F_{s i}(t)=K_{i} \cdot \Delta X_{i}(i=1,2)$, in which $\Delta X_{i}$ is the relative displacement increment of each SDOF structure, and
$K_{i}$ is the structural stiffness which relates to the yielding displacement $u_{y i}$ of each structure. When the SDOF structure is in elastic state during its motion, the stiffness of the structure is $K_{i}=m_{i} \omega_{i}^{2}$. When the displacement of the structure is greater than its yield displacement $u_{y i}$, the structure goes into the plastic phase, and the stiffness of the structure is $K_{i}=\alpha m_{i} \omega_{i}^{2}$, where $\alpha$ is the stiffness coefficient of the structure after yielding.

Dividing the two sides of equation (4) by $m_{2}$,

$$
\left\{\frac{m_{1}}{m_{2}} \ddot{X}_{1}(t)+2 \xi \frac{m_{1}}{m_{2}} \omega_{1} \dot{X}_{1}(t)+\frac{F_{s 1}(t)}{m_{2}}+\frac{F_{p 12}}{m_{2}}=-\frac{m_{1}}{m_{2}} \ddot{X}_{g}(t), \ddot{X}_{2}(t)+2 \xi \omega_{2} \dot{X}_{2}(t)+\frac{F_{s 2}(t)}{m_{2}}-\frac{F_{p 12}}{m_{2}}=-\ddot{X}_{g}(t) .\right.
$$

In order to convert equation (5) into dimensionless equation of motion, the physical quantity of the energy scale characterized by $l_{e}$ [9] $\left(l_{e}=a_{p} / \omega_{p}^{2}\right.$ with the dimensionless expression of [L]) is adopted in this paper, in which the mass $m_{2}$ of the right-side structure, the amplitude $a_{p}\left(\mathrm{~m} / \mathrm{s}^{2}\right)$, and circular frequency $\omega_{p}\left(s^{-1}\right)$ of sinusoidal excitation are chosen as the basic quantity. Then, the following transformation is done:

$$
\begin{aligned}
t & =\frac{\tau}{\omega_{p}} \\
X_{i}(t) & =x_{i}(\tau) \cdot l_{e}=\frac{x_{i}(\tau) \cdot a_{p}}{\omega_{p}^{2}}, \\
\dot{X}_{i}(t) & =\frac{\dot{x}_{i}(\tau) \cdot a_{p}}{\omega_{p}}, \\
X_{i}(t) & =\ddot{x}_{i}(\tau) \cdot a_{p},
\end{aligned}
$$

where $\tau$ is dimensionless movement time. $x_{i}(\tau), \dot{x}_{i}(\tau)$, and $\ddot{x}_{i}(\tau)(i=1,2)$ are the relative displacement, relative velocity, and relative acceleration of the dimensionless inelastic SDOF structures, respectively.

Substituting equations (6) into (5), the dimensionless equation of motion can be obtained:

$$
\left\{\frac{m_{1}}{m_{2}} \ddot{x}_{1}(\tau)+2 \xi \frac{m_{1}}{m_{2}} \frac{\omega_{1}}{\omega_{p}} \dot{x}_{1}(\tau)+\frac{F_{s 1}}{m_{2} a_{p}}+\frac{F_{p 12}}{m_{2} a_{p}}=-\frac{m_{1}}{m_{2}} \sin (\tau), \ddot{x}_{2}(\tau)+2 \xi \frac{\omega_{2}}{\omega_{p}} \dot{X}_{2}(t)+\frac{F_{s 2}}{m_{2} a_{p}}-\frac{F_{p 12}}{m_{2} a_{p}}=-\sin (\tau)\right.
$$

where $F_{s i} / m_{2} a_{p}$ represents dimensionless inelastic resistance and it is related to dimensionless structural stiffness $K_{i} / m_{2} \omega_{p}^{2}$ and dimensionless yield displacement $u_{y i} / l_{e}$. When the structure is in the elastic stage, the dimensionless stiffness of the structure is $K_{i} / m_{2} \omega_{p}^{2}=\left(m_{i} / m_{2}\right) \cdot\left(\omega_{i}^{2} / \omega_{p}^{2}\right)$, and when the structure is in the plastic stage, the dimensionless stiffness of the structure is $K_{i} / m_{2} \omega_{p}^{2}=\alpha \cdot\left(m_{i} / m_{2}\right) \cdot\left(\omega_{i}^{2} / \omega_{p}^{2}\right)$.
By using the improved Kelvin model, when the relative displacement $X$ exceeds the initial distance $d$, the pounding force is

$$
F_{p 12}= \begin{cases}\bar{k} \cdot \delta(t)+\bar{c} \cdot \dot{\delta}(t), & \delta(t)>d \\ 0, & \delta(t) \leq d\end{cases}
$$



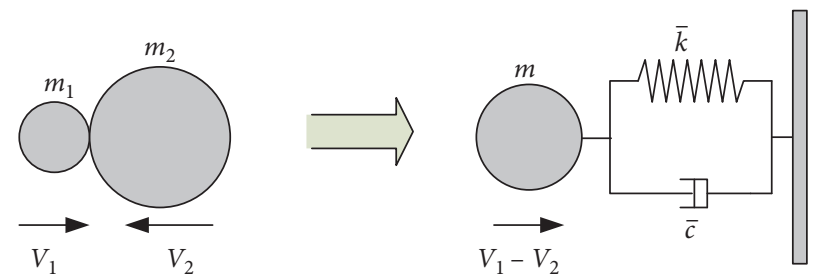

Figure 1: The improved Kelvin analytical model.

where $\quad \delta(t)=X_{1}-X_{2}-d, \quad \dot{\delta}(t)=\dot{X}_{1}-\dot{X}_{2}$, $\bar{k}=\bar{\omega}^{2} m_{1} m_{2} / m_{1}+m_{2}$, and $\bar{c}=3 \bar{k}(1-e) \cdot \delta(t) / 2 e\left(V_{1}-V_{2}\right)$. Substituting equations (6) into (8), when $x_{1}-x_{2}>d / l_{e}$, the two SDOF structures collide, and the expression of pounding force is as follows:

$$
F_{p 12}=\frac{m_{1} m_{2}}{m_{1}+m_{2}} \bar{\omega}^{2}\left(x_{1}-x_{2}-\frac{d}{l_{e}}\right) \cdot \frac{a_{p}}{\omega_{p}^{2}}+\frac{3(1-e)}{2 e\left(V_{1}-V_{2}\right)} \cdot \frac{m_{1} m_{2}}{m_{1}+m_{2}} \bar{\omega}^{2}\left(\dot{x}_{1}-\dot{x}_{2}\right) \cdot \frac{a_{p}}{\omega_{p}}\left(x_{1}-x_{2}-\frac{d}{l_{e}}\right) \cdot \frac{a_{p}}{\omega_{p}^{2}},
$$

when $x_{1}-x_{2}<d / l_{e}$, no pounding occurs; at this time, the expression of pounding force is as follows:

$$
F_{p 12}=0 \text {. }
$$

Equation (9a) is dimensionless and the dimensionless pounding force can be obtained when pounding occurs.

$$
\frac{F_{p 12}}{m_{2} a_{p}}=\frac{\left(m_{1} / m_{2}\right)}{\left(m_{1} / m_{2}\right)+1}\left[\left(\frac{\bar{\omega}}{\omega_{p}}\right)^{2} \cdot\left(x_{1}-x_{2}-\frac{d}{l_{e}}\right)+\frac{3(1-e)}{2 e} \cdot \frac{1}{v_{1}-v_{2}} \cdot\left(\frac{\bar{\omega}}{\omega_{p}}\right)^{2} \cdot\left(\dot{x}_{1}-\dot{x}_{2}\right) \cdot\left(x_{1}-x_{2}-\frac{d}{l_{e}}\right)\right] \text {, }
$$

where $v_{1}$ and $v_{2}$ are dimensionless velocities $\left(v_{i}=V_{i} \cdot\left(\omega_{p} / a_{p}\right)\right)$ when pounding occurs between left and right structures, respectively.

The dimensionless equations of motion of two inelastic SDOF structures can be obtained by combining equations (7) and (10).

\subsection{The Dimensionless Equation of Motion Based on the} Buckingham $\Pi$ Theorem. According to the $\Pi$ theorem, the pounding equation of motion between two inelastic SDOF structures obtained above and the dimensionless parameters which characterize poundings of adjacent structures are presented in literature [16], and the parameters that characterize the pounding response of two inelastic SDOF structures under sinusoidal excitation are peak displacement $X_{\max }$ and peak velocity $\dot{X}_{\max }$ of the structures with pounding. The parameters controlling the reaction are the mass $m_{1}$ and $m_{2}$, the angular frequency $\omega_{1}$ and $\omega_{2}$, the yield displacement $u_{y 1}$ and $u_{y 2}$, the damping ratio $\xi$, the initial spacing $d$ of the two inelastic SDOF structures and the stiffness coefficient $\alpha$ of the structure after yielding, the angular frequency $\bar{\omega}$ and recovery coefficient $e$ of the contact element, and the amplitude $a_{p}$ and angular frequency $\omega_{p}$ of the sinusoidal excitation.
Through the $\Pi$ theorem, the pounding response function of the two inelastic SDOF structures can be expressed as follows:

$$
\left.\begin{array}{c}
X_{\max } \\
\dot{X}_{\max }
\end{array}\right\}=f\left(m_{1}, m_{2}, \omega_{1}, \omega_{2}, u_{y 1}, u_{y 2}, \xi, \alpha, \bar{\omega}, e, d, a_{p}, \omega_{p}\right)
$$

According to equation (11), there are 14 variables in the equation, among which there are three basic dimensions: mass [M], length [L], and time [T]. According to the $\Pi$ theorem, 11 independent dimensionless parameters can be obtained. In Section 2.2, the mass $m_{2}$ of the right-side SDOF structure, the amplitude $a_{p}$, and angular frequency $\omega_{p}$ of the sinusoidal excitation acceleration are selected as the basic variables. The equation of motion of two inelastic SDOF is dimensionless, and equation (11) can be changed by

$$
\left.\begin{array}{c}
\frac{X_{\max } \omega_{p}^{2}}{a_{p}} \\
\frac{\dot{X}_{\max } \omega_{p}}{a_{p}}
\end{array}\right\}=\phi\left(\frac{m_{1}}{m_{2}}, \frac{\omega_{1}}{\omega_{p}}, \frac{\omega_{2}}{\omega_{p}}, \frac{u_{y 1} \omega_{p}^{2}}{a_{p}}, \frac{u_{y 2} \omega_{p}^{2}}{a_{p}}, \xi, \alpha, \frac{\bar{\omega}}{\omega_{p}}, e, \frac{d \omega_{p}^{2}}{a_{p}}\right),
$$




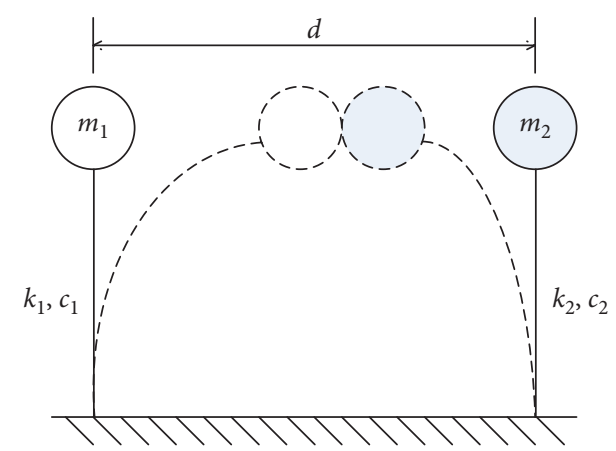

FIgURE 2: Pounding model of two inelastic SDOF systems.

where

$$
\left\{\begin{array}{l}
\Pi_{u}=\frac{X_{\max } \omega_{p}^{2}}{a_{p}} \\
\Pi_{v}=\frac{\dot{X}_{\max } \omega_{p}}{a_{p}} \\
\Pi_{m}=\frac{m_{1}}{m_{2}}, \\
\Pi_{\omega 1}=\frac{\omega_{1}}{\omega_{p}} \\
\Pi_{\omega 2}=\frac{\omega_{2}}{\omega_{p}} \\
\Pi_{u y 1}=\frac{u_{y 1} \omega_{p}^{2}}{a_{p}} \\
\Pi_{\xi}=\alpha, \\
\Pi_{u y 2}=\frac{u_{y 2} \omega_{p}^{2}}{a_{p}}
\end{array}\right.
$$

Accordingly, equation (12) can be rewritten as

$$
\left.\begin{array}{c}
\Pi_{u} \\
\Pi_{v}
\end{array}\right\}=\phi\left(\Pi_{m}, \Pi_{\omega 1}, \Pi_{\omega 2}, \Pi_{u y 1}, \Pi_{u y 2}, \Pi_{\xi}, \Pi_{\alpha}, \Pi_{\omega \mathrm{con}}, \Pi_{e}, \Pi_{d}\right),
$$

where $\Pi_{m}=m_{1} / m_{2}$ is the mass ratio of the left-side SDOF structure to the right-side SDOF structure. $\Pi_{\omega i}=\omega_{i} / \omega_{p},(i=$ $1,2)$ is the ratio of the angular frequency of each inelastic SDOF structure to the angular frequency of the sinusoidal excitation, that is, the dimensionless angular frequency of each structure. $\Pi_{u y i}=u_{y i} \omega_{p}^{2} / a_{p}(i=1,2)$ is the ratio of yield displacement to the excitation energy scale $l_{e}=a_{p} / \omega_{p}^{2}$ of each structure, that is, dimensionless yield displacement. $\Pi_{u y i}$ and $\Pi_{\alpha}$ are the parameters characterizing structural inelasticity. $\Pi_{\omega \mathrm{con}}, \Pi_{d}$, and $\Pi_{e}$ are parameters characterizing the pounding characteristics, where $\Pi_{\omega \mathrm{con}}=\bar{\omega} / \omega_{p}$ represents the ratio of angular frequency of contact element to the sinusoidal excitation, that is, the angular frequency of dimensionless contact element.

\section{Numerical Solution of Pounding Response of Two Inelastic SDOF Structures}

In order to study the dimensionless pounding response of two inelastic SDOF structures, Newmark- $\beta$ method [28] is used to solve equation (7), where the parameters are taken as $\gamma=1 / 2$ and $\beta=1 / 4$. The time step is $\Delta \tau=0.001$. Previous scholars [24] divided the pounding reaction into three spectral regions (amplification region, inhibition region, and no obvious influence region) in the study of the pounding response between a single oscillator and a rigid barrier. Similarly, in order to study the pounding response of two inelastic SDOF structures in different frequency regions (the amplification region in the first spectral region and the suppression region in the second spectral region), two sets of different parameters are considered.

Parameter set 1:

$$
\left\{\begin{aligned}
\Pi_{m} & =\frac{m_{1}}{m_{2}}=0.5, \\
\Pi_{\omega 1} & =\frac{\omega_{1}}{\omega_{p}}=1.05, \\
\Pi_{\omega 2} & =\frac{\omega_{2}}{\omega_{p}}=2.36, \\
\Pi_{u y 1} & =0.9, \\
\Pi_{u y 2} & =0.8, \\
\Pi_{\xi} & =0.05, \\
\Pi_{\alpha} & =0.1, \\
\Pi_{\omega \mathrm{con}} & =50, \\
\Pi_{e} & =0.4
\end{aligned}\right.
$$


Parameter set 2:

$$
\left\{\begin{array}{l}
\Pi_{m}=\frac{m_{1}}{m_{2}}=0.5, \\
\Pi_{\omega 1}=\frac{\omega_{1}}{\omega_{p}}=1.5, \\
\Pi_{\omega 2}=\frac{\omega_{2}}{\omega_{p}}=3.375, \\
\Pi_{u y 1}=0.9, \\
\Pi_{u y 2}=0.8, \\
\Pi_{\xi}=0.05, \\
\Pi_{\alpha}=0.1, \\
\Pi_{\omega \mathrm{con}}=50, \\
\Pi_{e}=0.4
\end{array}\right.
$$

From the above two parameter sets, it can be seen that the only differences between the two groups of parameters are the dimensionless angular frequencies $\Pi_{\omega 1}$ and $\Pi_{\omega 2}$ of each SDOF structure. However, the ratios of dimensionless angular frequencies of the two SDOF structures are equal; that is, $\Pi_{\omega 2} / \Pi_{\omega 1}=2.36 / 1.05=3.375 / 1.5=2.25$. These two sets of parameters correspond to the pounding reaction of the first spectral region (amplification region) and the second spectral region (suppression region) proposed by the former scholars [24].

3.1. Dimensionless Time-History Responses. The dynamic equation of motion of equation (7) is solved numerically by using MATLAB software. Figure 3 shows the dimensionless displacement response, pounding force response, and their respective story shear drift loop of the two adjacent inelastic SDOF structures with parameter set 1 .

Figures 3(a) and 3(b) show the displacement and the pounding force histories of two inelastic SDOF structures under sinusoidal excitation, respectively, where the relative displacement response time-history curves of the left-side structure coincide with those of the right-side structure, and the pounding between the two structures occurs as shown in Figure 3(a). Therefore, as can be seen from Figure 3(a), a total of nine poundings have taken place between the two structures. The pounding force shown in Figure 3(b) also has nine sudden changes, which coincides with the number of poundings judged in Figure 3(a). In addition, Figure 3 also shows that, after pounding, the positive displacement of the left-side structure (the structure with smaller mass and stiffness) is significantly suppressed, but there is a very obvious rebound in the reverse direction, resulting in a very large negative displacement, and the peak displacement response increases after pounding. As for the right-side structure, both the positive and negative displacement responses increase greatly after pounding. This phenomenon is clearly plotted by Figures 4(a) and 4(c) .

Figure 3(c) shows the story shear drift loop of two adjacent inelastic SDOF structures with parameter set 1 , which is a hysteretic curve formed by coupling the displacement time history curve and collision force time history curve into one graph. As can be seen from Figures 3(c) and 3(d), the left-side structure (with smaller mass and stiffness) obviously enters the plastic stage during the whole process, and the hysteretic curve of the right-side structure (with larger mass and stiffness) is a straight line, which indicates that it has been in the elastic stage.

Figures 4 and 5 show the dimensionless displacement and velocity histories of the left-side and right-side structures under different parameters when pounding and no pounding occurs. The effect of pounding on structural response under different parameters is studied. As shown in Figure 4(a), the positive displacement of the left-side structure with smaller mass and stiffness is greatly suppressed, while the negative displacement is obviously increased, resulting in larger negative displacement. As for the right-side structures with larger mass and stiffness, as shown in Figure 4(c), both positive and negative displacements increase. In addition, the pounding also has an obvious effect on the velocity response of the two structures. From the velocity histories shown in Figures 4(b) and 4(d), it can be seen that, after pounding, the velocity of the two structures changes dramatically. The velocity response of the left-side structure decreases, while that of the right-side structure increases. Therefore, one of the basic characteristics of pounding is the rapid change of velocity.

Figure 5 shows that the displacement response of the leftside structure decreases after pounding, while the displacement and velocity response of the right-side structure increase under the condition of parameter set 2, which is different from the response of the SDOF structures in Figure 4 . The reason for these differences is that the dimensionless angular frequencies $\Pi_{\omega 1}$ and $\Pi_{\omega 2}$ of the two structures in parameter sets 1 and 2 are different; that is, the stiffness of the structure is different, so the impact of pounding on the structural response is closely related to the stiffness of the structure itself.

In addition, the displacement and velocity histories obtained by improved Kelvin model and Kelvin model are, respectively, compared in Figures 4 and 5. From the displacement time-history curves in Figures 4(a), 4(c), 5(a), and $5(c)$, it can be seen that the two curves basically coincide. However, as can be seen from the enlarged part of the velocity time-history curves in Figures 4(b), 4(d), 5(b), and $5(\mathrm{~d})$, when the Kelvin model is used to simulate the pounding process, negative tension will appear in the 


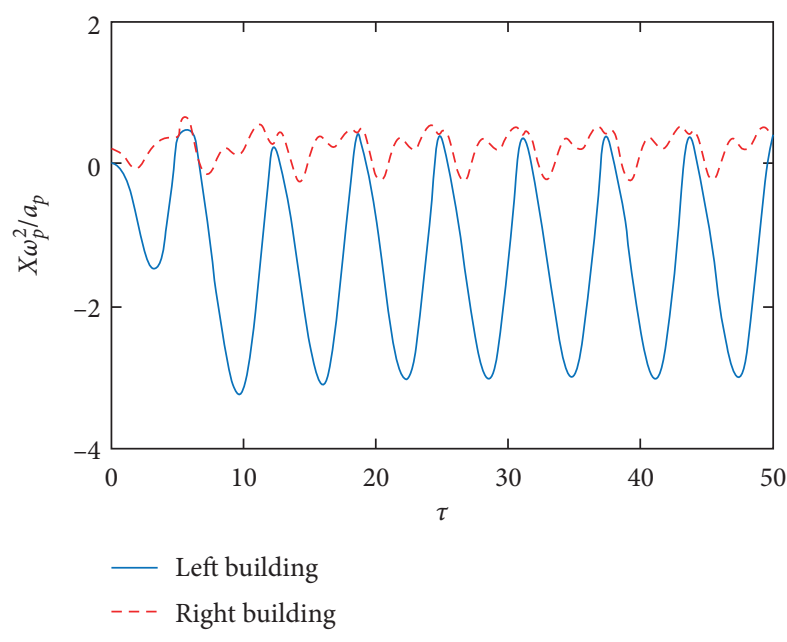

(a)

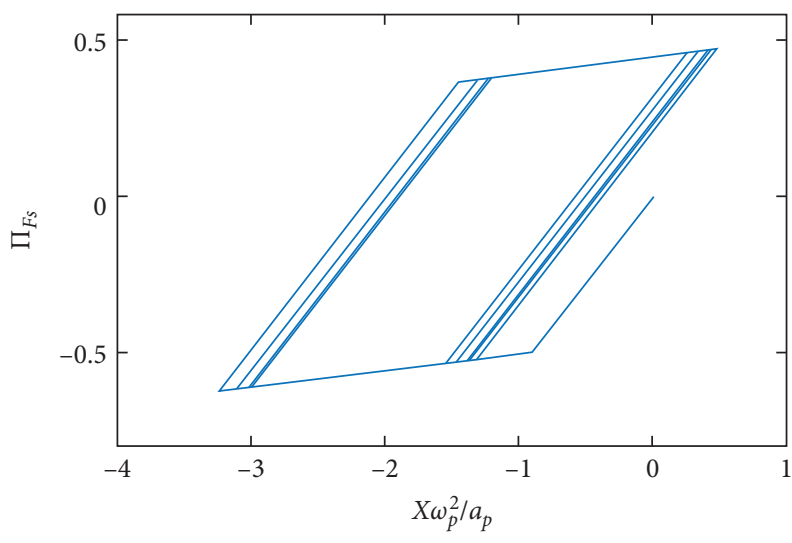

(c)

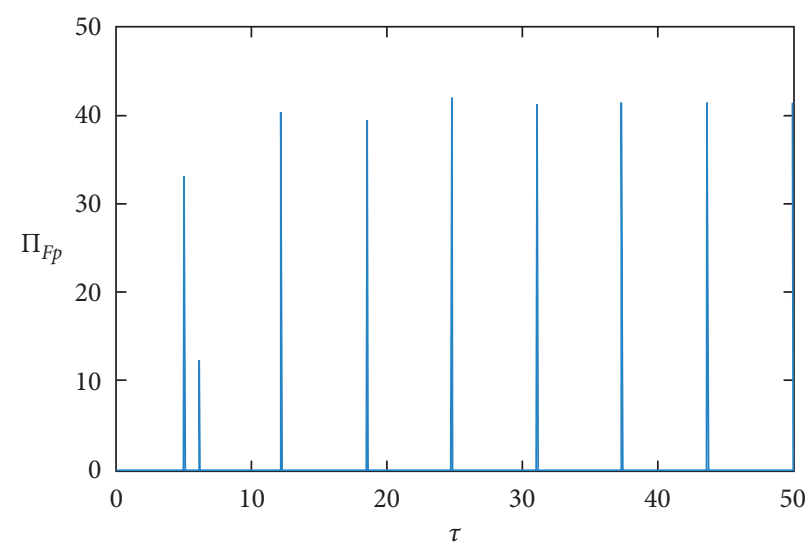

(b)

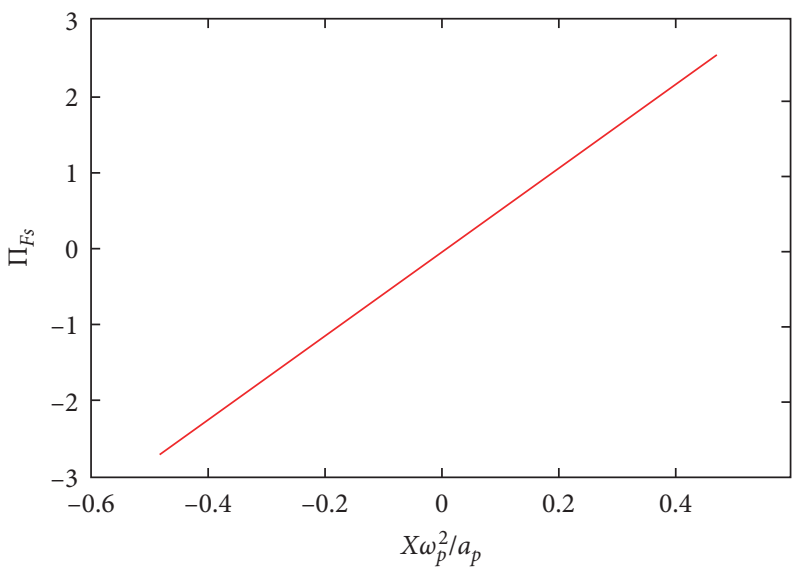

(d)

FIgURE 3: Time-histories and force-drift loops of two inelastic SDOF structures with parameter set 1. (a) Displacement histories. (b) Pounding force histories. (c) The story shear force-drift loop for left-side structure. (d) The story shear force-drift loop for right-side structure.

rebounding stage, which results in the velocity histories first increasing and then decreasing. However, the response calculated by the improved Kelvin model can avoid this phenomenon, which verifies the superiority of the improved Kelvin model. This situation is well illustrated by the pounding force time-history curves obtained by using the improved Kelvin model and Kelvin model under parameter set 1 as shown in Figure 6. When pounding occurs, the pounding force curves obtained by Kelvin model simulating pounding process have negative values, while the curves obtained by improved Kelvin model have no negative tension. Therefore, the improved Kelvin model overcomes the theoretical defects of the Kelvin model as plotted in Figure 6, which can reasonably reflect the physical nature of poundings.

3.2. The Significance of Applying the Dimensional Analysis. When using dimensional method to analyze the pounding response of the adjacent structures, the self-similarity of the structures can be well explained. Figure 7 shows the maximum displacement and velocity response curves of two adjacent inelastic SDOF structures under different excitation amplitudes using the improved Kelvin model to simulate the pounding force. In this case, assuming $\Pi_{\omega 2}=\mu \Pi_{\omega 1}, \mu=2.25$, and the other parameters are the same as those of equation (15).

In Figures $7(\mathrm{a})$ and $7(\mathrm{c})$, under three different intensity levels $\left(a_{p}=0.2 g, 0.5 g, 0.8 g\right)$ of excitation, three different peak velocity curves with and without pounding with real physical units are obtained, respectively. However, the velocity curves are reduced into a single master curve no matter for the case of pounding or no pounding when plotted in terms of the dimensionless $\Pi$-terms as can be seen in Figures 8(b) and 8(d). This observation indicates that the impact response of two inelastic SDOF structures is not affected by the excitation peak acceleration when the dimensionless parameter is used; that is, the impact response of two inelastic SDOF structures has its self-similarity [19].

In addition, it can also be seen from Figure 7(b) that, for the left-side structure (with smaller mass and stiffness), when the dimensionless frequency $\Pi_{\omega 1}$ is small 


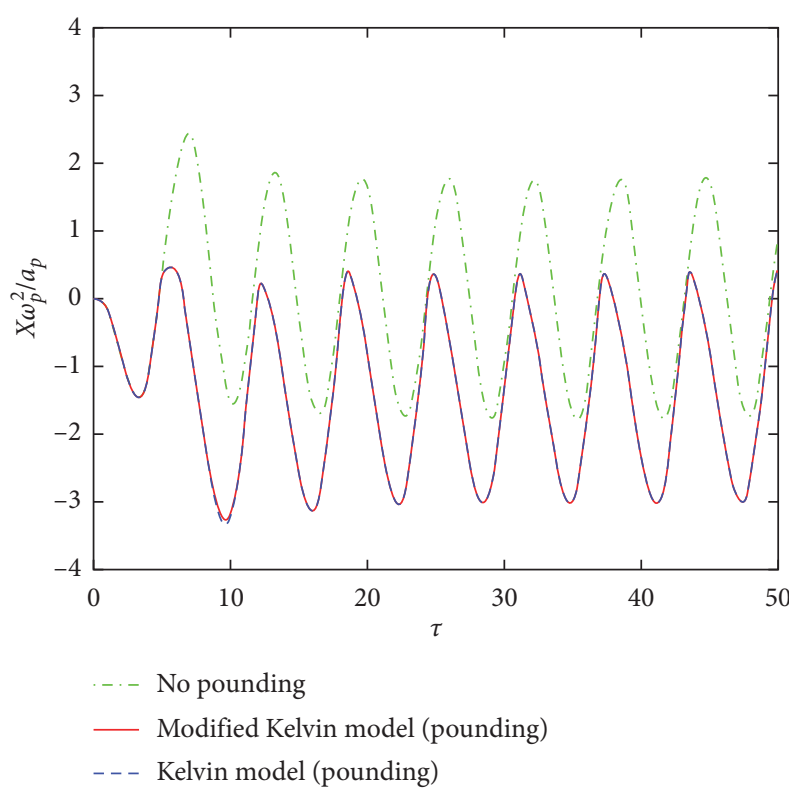

(a)

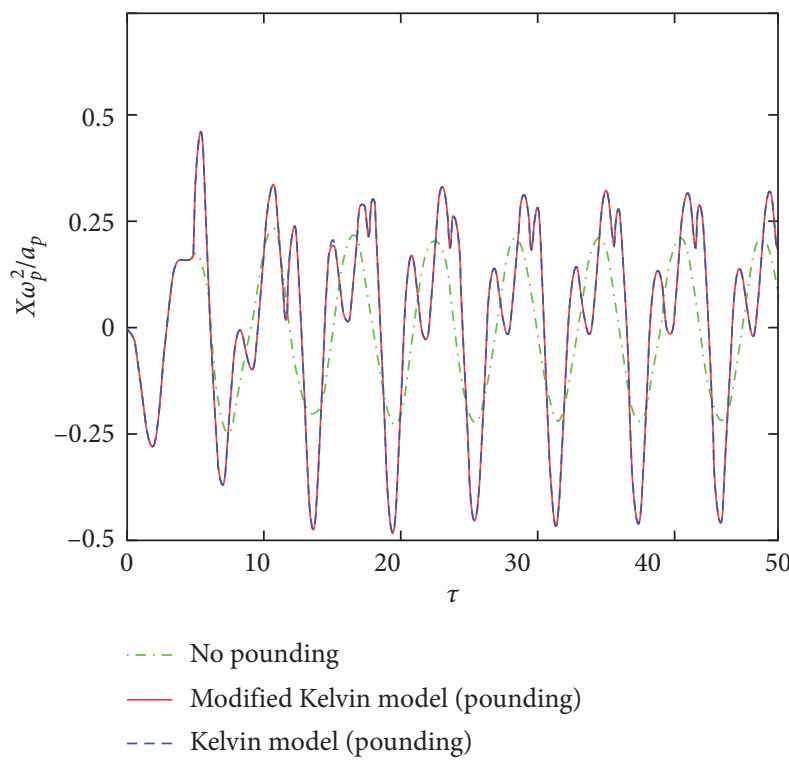

(c)

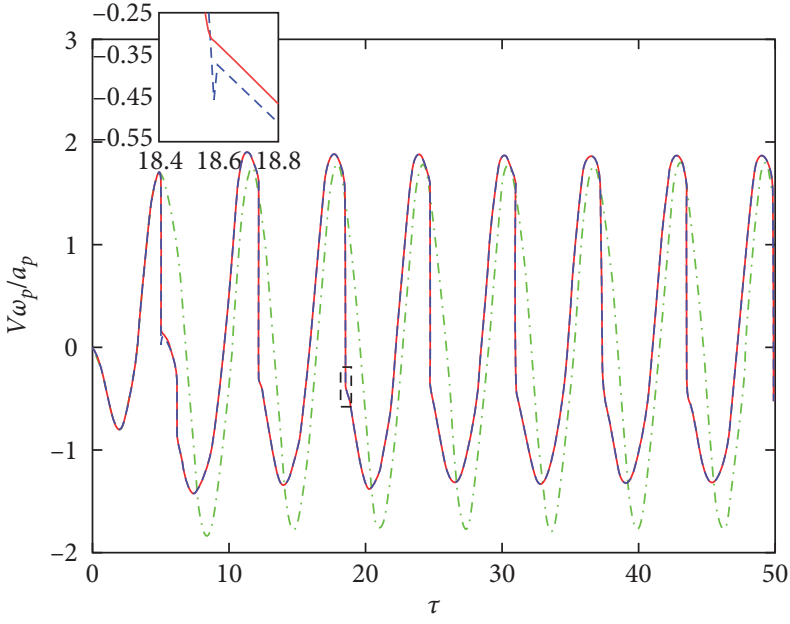

(b)

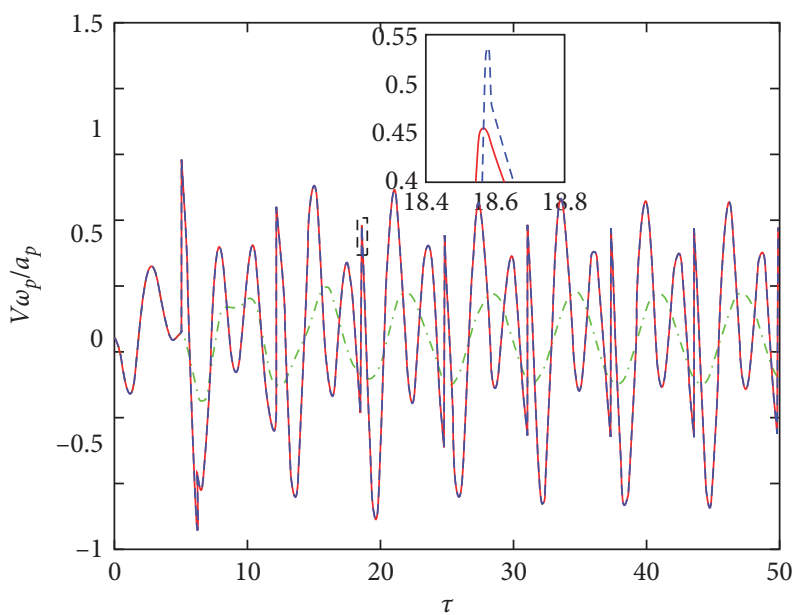

(d)

FIGURE 4: Time-history responses of two inelastic SDOF structures under different impact elements with parameter set 1. (a) Displacement histories of left-side structure. (b) Velocity histories of left-side structure. (c) Displacement histories of right-side structure. (d) Velocity histories of right-side structure.

$\left(0.5<\Pi_{\omega 1}<1.25\right)$, the pounding will increase its displacement response, when the dimensionless frequency $\Pi_{\omega 1}$ is large $\left(1.25<\Pi_{\omega 1}<1.75\right)$, the pounding will reduce the displacement response, and when the dimensionless frequency continues to increase to a certain extent $\left(\Pi_{\omega 1}>1.75\right)$, the peak displacement curves of pounding and no pounding of the structure coincide basically, which shows that the pounding response of this stage has little influence on the displacement response of the structure. This corresponds to the three spectral regions (the amplification region of the first spectral region, the suppression region of the second spectral region, and the noninfluence region of the third spectral region) obtained by previous scholars [19] who studied the impact of pounding between an elastic single oscillator and a rigid barrier on the structural response. For the pounding response of two inelastic SDOF structures in this paper, the division of three spectral regions is shown in Figure 7(b). The dimensionless peak displacement responses of right-side structures (with larger mass and stiffness) increase after pounding, and there is no spectral division as shown in Figure 7(d). For the dimensionless frequencies $\Pi_{\omega 1}$ of the two sets of parameters (equations (14) and (15)), the dimensionless frequency $\Pi_{\omega 1}$ of the first 


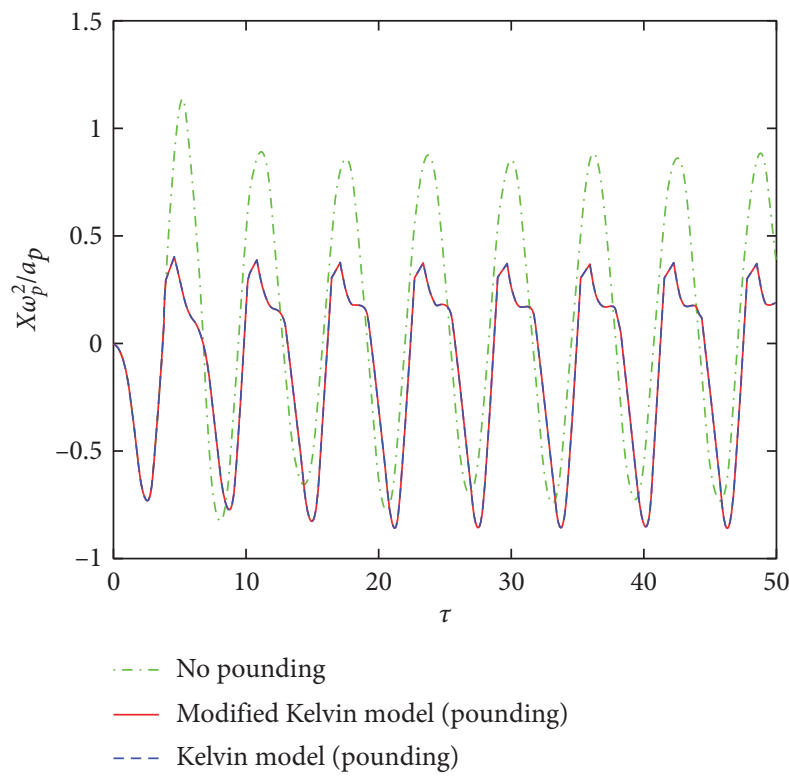

(a)

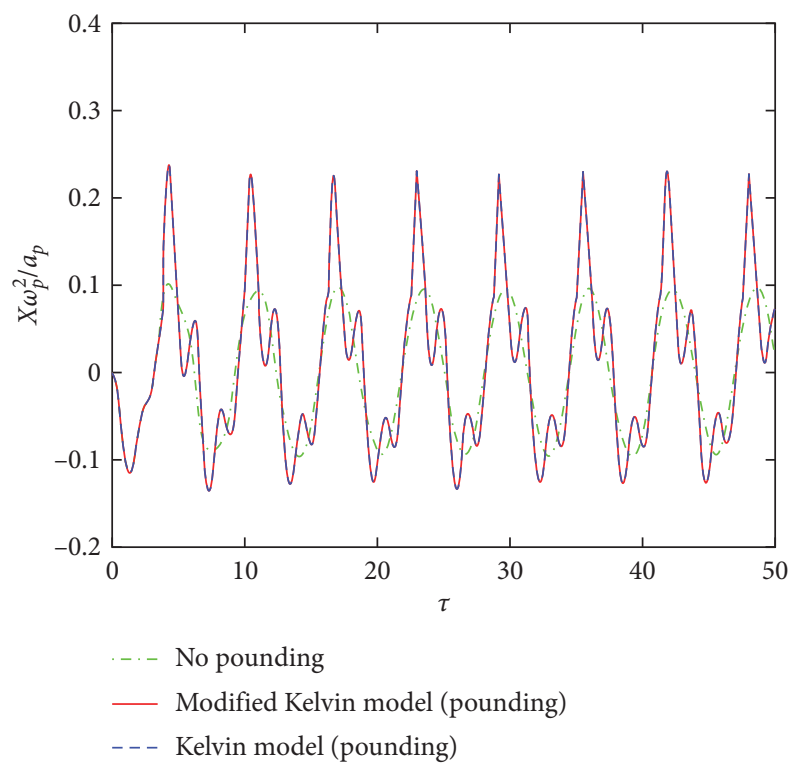

(c)

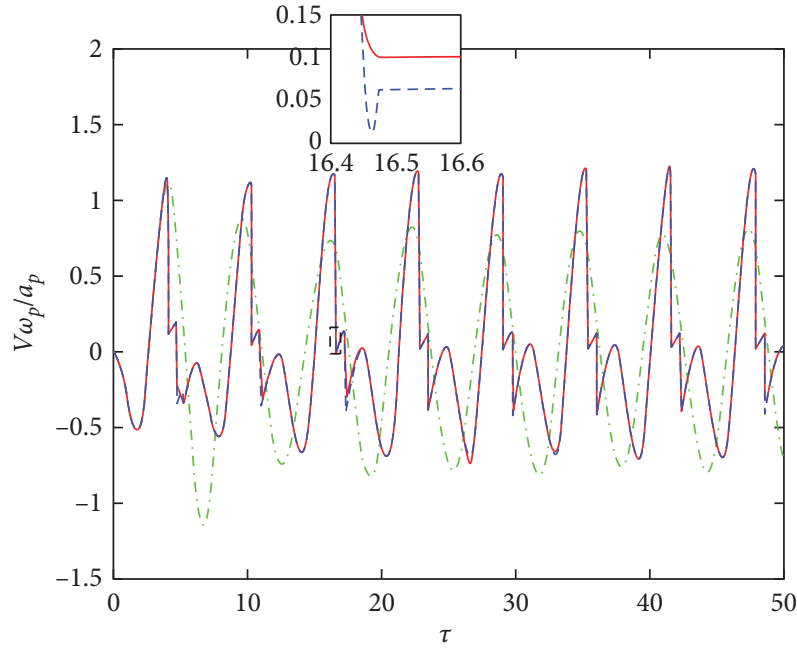

(b)

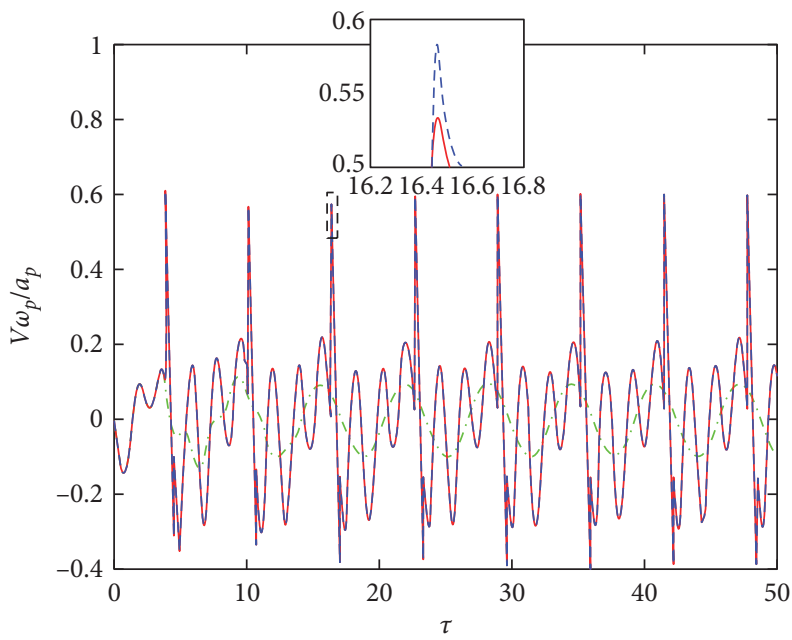

(d)

Figure 5: Time-history responses of two inelastic SDOF structures under different impact elements with parameter set 2. (a) Displacement histories of left-side structure. (b) Velocity histories of left-side structure. (c) Displacement histories of right-side structure. (d) Velocity histories of right-side structure.

parameter set is in the first spectral region, and the dimensionless frequency $\Pi_{\omega 1}$ of the second parameter set is in the second spectral region, and the impact of the pounding reaction is exactly the same as the division of the spectral region, which proves the correctness of the division of the three spectral regions.

\section{Parametric Analysis of Pounding}

Previous scholars [24] have made parametric analysis on the pounding between elastic single oscillator structure and rigid barrier and studied the influence of the parameters of impact elements on the pounding response of structures. It was found that the peak displacement and peak velocity of the single oscillator are not affected by the contact stiffness during the whole pounding process. Therefore, in this paper, the effects of structural parameters on pounding reaction are mainly studied, including the influence of mass ratio $\Pi_{m}$, frequency ratio $\mu\left(\mu=\Pi_{\omega 2} / \Pi_{\omega 1}\right)$, and structural spacing $\Pi_{d}$ of the two adjacent inelastic SDOF structures on the pounding response.

4.1. Effects of Mass Ratio $\Pi_{m}$. Figure 9 shows the dimensionless maximum displacement $\Pi_{u}$ and the maximum 


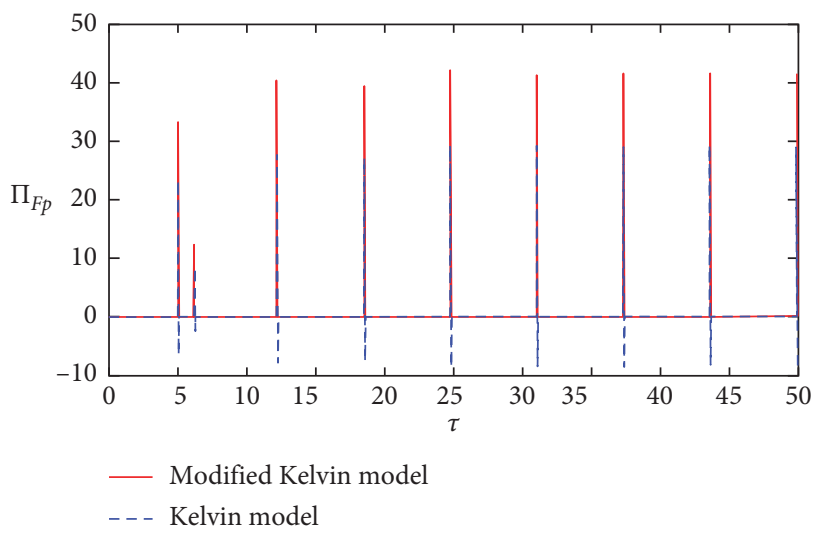

FIGURE 6: The pounding force histories of two inelastic SDOF structures under parameter set 1 using improved Kelvin model and Kelvin model.

velocity $\Pi_{v}$ versus the angular frequency ratio $\Pi_{\omega 1}$ for various mass ratios $\Pi_{m}$, respectively. Three different mass ratios $\left(\Pi_{m}=0.1, \Pi_{m}=0.5\right.$, and $\left.\Pi_{m}=0.9\right)$ are selected here. The larger the mass ratio is, the smaller difference between the left-side and right-side structures is.

Figures 9(a) and 9(b) show that when $\Pi_{\omega 1}<0.6$, the three curves coincide, which shows that there is no pounding between the two inelastic adjacent SDOF structures, and the selection of different mass ratios has no effect on the displacement and velocity responses of the structures. With the increase of $\Pi_{\omega 1}$, when $0.6 \leq \Pi_{\omega 1}<1.1$ and $\Pi_{m}=0.9$, the dimensionless peak displacement and velocity of the left-side (with smaller mass and stiffness) structure are obviously smaller than those corresponding to $\Pi_{m}=0.1$ and $\Pi_{m}=0.5$, which shows that when the mass difference between adjacent structures is large, the structural response will be larger. At this time, the displacement and velocity response of the left-side structure will decrease with the increase of mass ratio. When $1.1 \leq \Pi_{\omega 1}<1.4$ and $\Pi_{m}=0.9$, the dimensionless peak displacement and velocity of the left-side structure are obviously larger than those corresponding to $\Pi_{m}=0.1$ and $\Pi_{m}=0.5$. At this time, the displacement and velocity responses of the left-side structure increase with the increase of mass ratio. When $\Pi_{\omega 1}$ continues to increase, the variety of mass ratio $\Pi_{m}$ has little effect on the displacement and velocity responses of the left-side structure.

Considering the three spectral regions, the influence of mass ratio $\Pi_{m}$ on the displacement and velocity response of the left-side structure (both mass and stiffness are small) can be corresponding to the three spectral regions. In the first spectral region (amplification region), the pounding will amplify the displacement and velocity response of the structure. In this spectral region, the displacement and velocity responses of the structure decrease obviously with the increase of mass ratio $\Pi_{m}$, which indicates that the amplification effect of pounding on the structural response decreases with the increase of mass ratio $\Pi_{m}$. In the second spectral region (suppression region), the pounding will reduce the displacement and velocity responses of the structure. In this spectral region, the displacement and velocity responses of the structure increase with the increase of mass ratio $\Pi_{m}$, which indicates that the restraint effect of pounding on the structural response decreases with the increase of mass ratio $\Pi_{m}$. In the third spectral region (no influence region), the displacement and velocity responses of the structure are basically not affected by the pounding effect and the variety of mass ratio $\Pi_{m}$.

In summary, when the mass ratio $\Pi_{m}$ increases (the difference of mass characteristics between left and right structures decreases), the impact of pounding on displacement and velocity responses (including magnification and suppression) of the left-side structure (both mass and stiffness are small) is also significantly reduced.

For the right-side structure with larger mass and stiffness, it can be seen from Figures $9(\mathrm{c})$ and $9(\mathrm{~d})$ that when $\Pi_{\omega 1}<0.6$, the three curves coincide, there is no pounding during this stage, and the change of mass ratio $\Pi_{m}$ has no effect on the response of the structure. When $0.6 \leq \Pi_{\omega 1}<2.1$, the adjacent structures collide, and the pounding magnifies the reaction of the right-side structure. The value of the curve corresponding to $\Pi_{m}=0.9$ is obviously larger than that corresponding to $\Pi_{m}=0.1$ and $\Pi_{m}=0.5$. The results show that, with the increase of mass ratio $\Pi_{m}$, the displacement and velocity responses of the structure increase, and the amplification effect of pounding on the right-side of the structure also increases. When the dimensionless frequency $\Pi_{\omega 1}$ continues to increase, the stiffness of the structure is larger and the impact effect on the response of the structures is smaller. When $\Pi_{\omega 1}>2.5$, the displacement of the structure tends to zero and no pounding occurs. As can be seen from Figures 9(c) and 9(d), the three curves with different mass ratios $\Pi_{m}$ coincide, which shows that the change of mass ratio $\Pi_{m}$ has no effect on the displacement and velocity response of the right-side structure.

4.2. Effects of Structural Frequency Ratio $\mu$. Figure 10 shows the response curves of dimensionless peak displacement $\Pi_{u}$ 


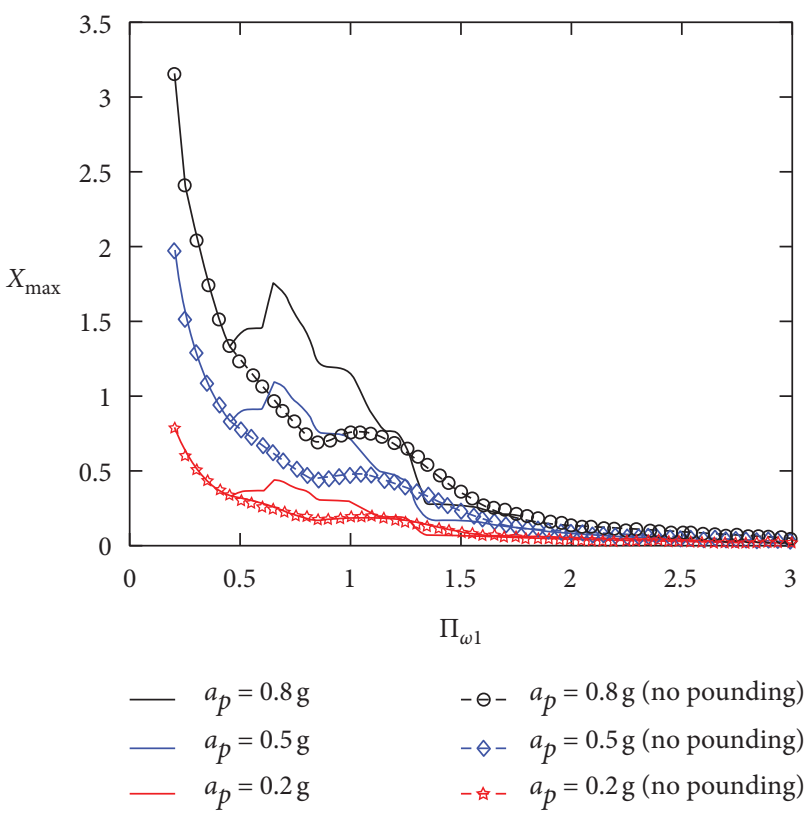

(a)

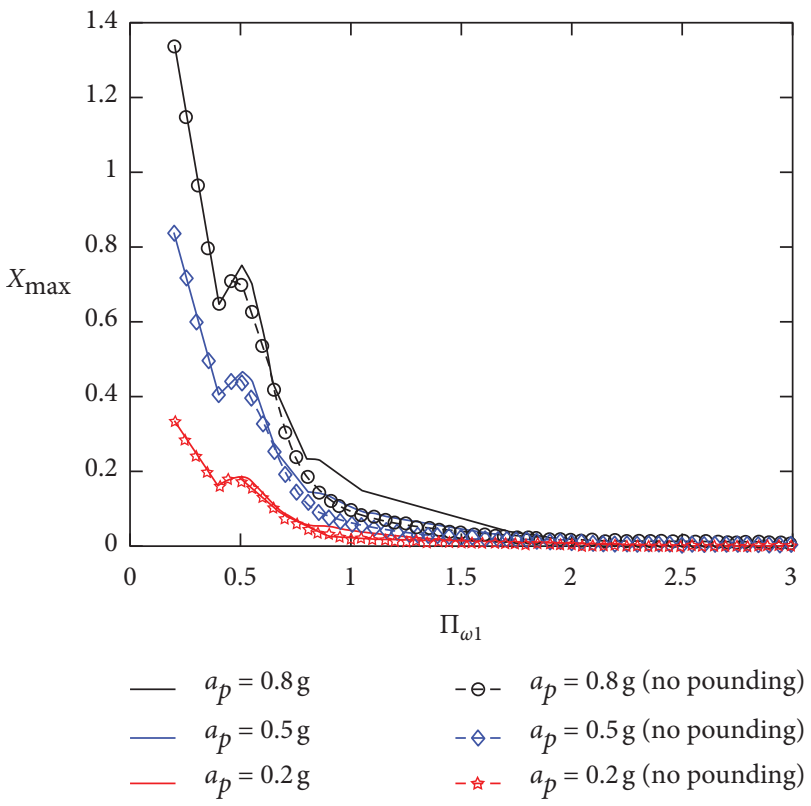

(c)

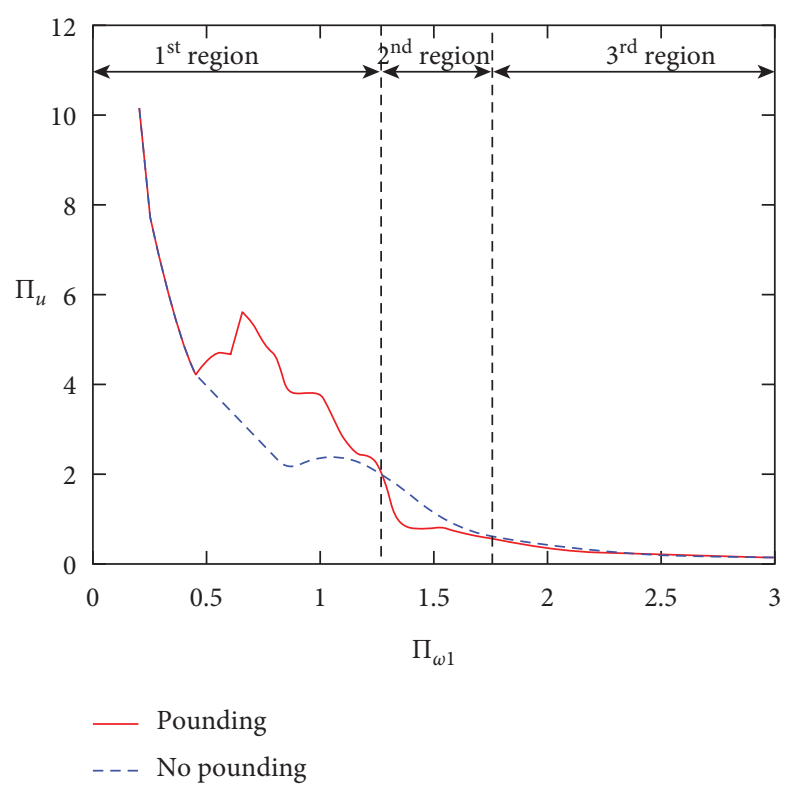

(b)

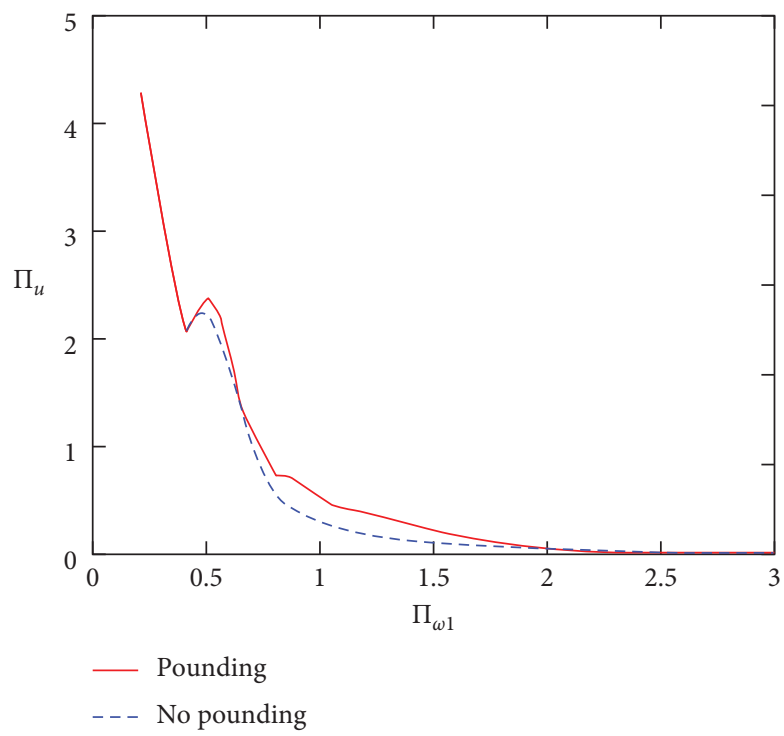

(d)

Figure 7: Dimensional and dimensionless peak displacement of the two inelastic SDOF structures under different amplitudes of acceleration. (a) Dimensional displacement of left-side structure. (b) Dimensionless displacement of left-side structure. (c) Dimensional displacement of right-side structure. (d) Dimensionless displacement of right-side structure.

and dimensionless peak velocity $\Pi_{v}$ of adjacent inelastic SDOF structures corresponding to different dimensionless angular frequencies $\Pi_{\omega 1}$ of structures under different structural frequency ratios $\mu$. Three different frequency ratios $\mu$ are selected here $(\mu=1.5, \mu=4$, and $\mu=10)$. The larger the frequency ratio $\mu$ is, the greater the stiffness difference between the left-side and right-side structure is, and the stiffness of the right-side structure is much larger than that of the left-side structure.

As shown in Figures 10(a) and 10(b), when $\Pi_{\omega 1}<0.9$, the left-side structure (with smaller mass and stiffness) is obviously affected by the variation of frequency ratio $\mu$. The displacement response value of the curve corresponding to frequency ratio $\mu=4$ is larger than that of the curves corresponding to $\mu=1.5$ and $\mu=10$. This may be due to the fact that when $\Pi_{\omega 1}<0.9$, the left-side structure is in the first spectral region (amplification region), and the displacement response of the left-side structure will increase as pounding occurs. When the frequency ratio of the structure increases from 1.5 to 4 , the difference of dynamic performance between the two adjacent structures increases gradually, and the pounding between the 


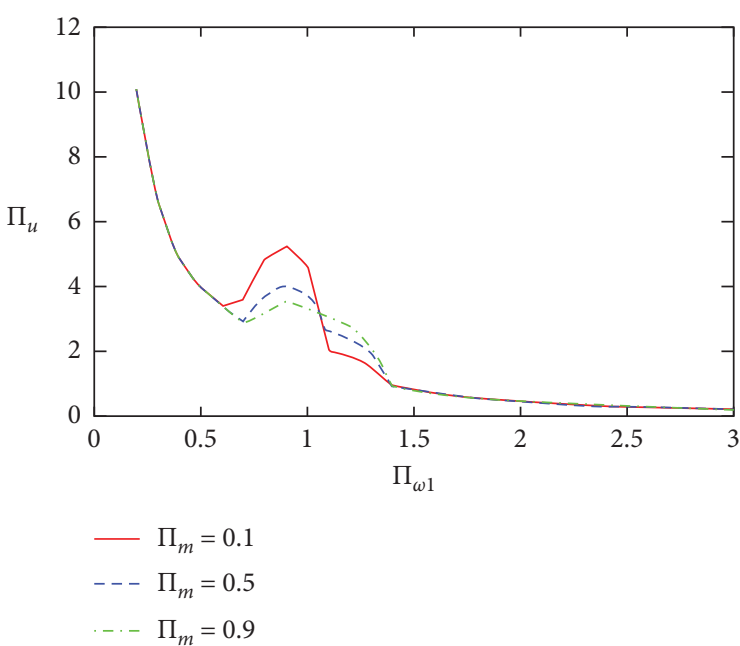

(a)

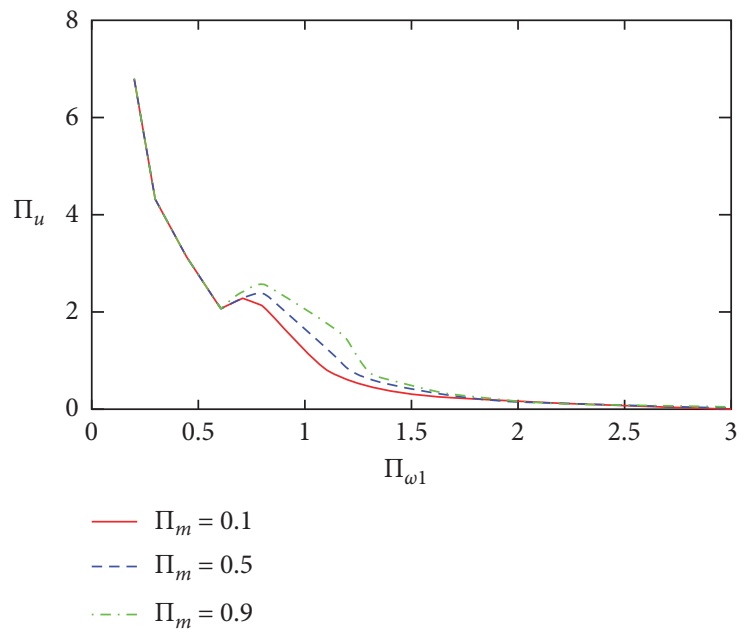

(c)

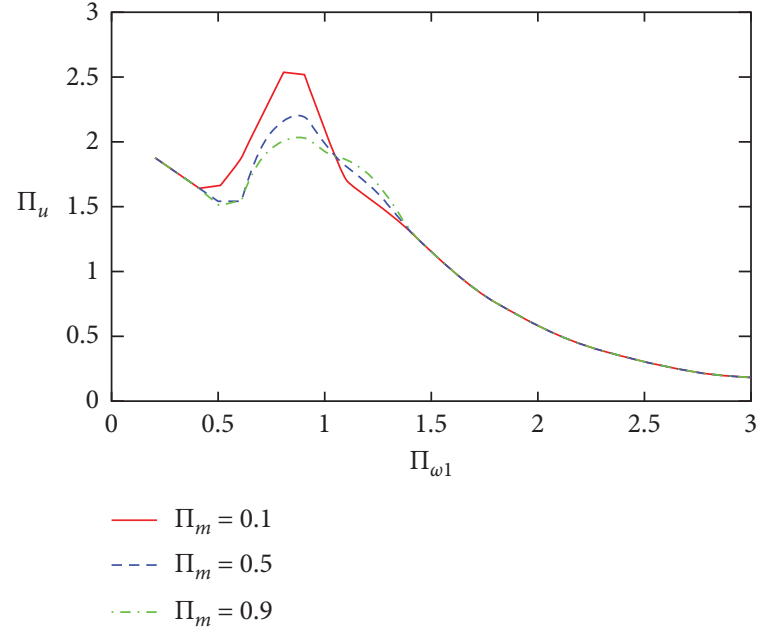

(b)

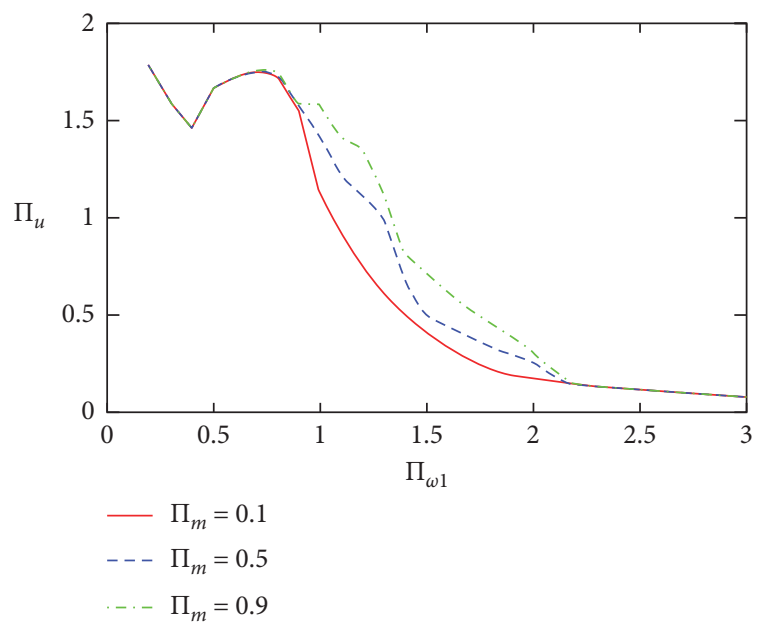

(d)

FiguRE 8: Maximum dimensionless displacement $\Pi_{u}$ and velocity $\Pi_{v}$ versus $\Pi_{\omega 1}$ for various structural spacing $\Pi_{d}\left(\Pi_{m}=0.5, \Pi_{\xi}=0.05\right.$, $\Pi_{u y 1}=0.9, \Pi_{u y 2}=0.8, \Pi_{u y 1}=0.9, \mu=1.5, \Pi_{\alpha}=0.1, \Pi_{\omega \mathrm{con}}=50$, and $\Pi_{r}=0.4$ ). (a) Maximum dimensionless displacement $\Pi_{u}$ of left-side structure. (b) Maximum dimensionless velocity $\Pi_{v}$ of left-side structure. (c) Maximum dimensionless displacement $\Pi_{u}$ of right-side structure. (d) Maximum dimensionless velocity $\Pi_{v}$ of right-side structure.

two structures increases, which enhances the magnification of the displacement response of the left-side structure. Therefore, the displacement response of the left-side structure increases with the increase of frequency ratio $\mu$. When the frequency ratio $\mu$ of the structure continues to increase from 4 to 10 , the stiffness of the right-side structure is very large, and the vibration amplitude of the structure under sinusoidal excitation decreases, so that the pounding between adjacent structures decreases accordingly. Therefore, the magnification of the pounding effect on the left-side structure decreases, resulting in a decrease in the displacement response of the left-side structure with the increase of frequency ratio $\mu$.

In the second spectral region, the influence of frequency ratio $\mu$ on the displacement response of the left-side structure is not obvious. For the right-side structure with larger mass and stiffness, the dimensionless peak displacement and velocity response curves are shown in Figures 10(c) and 10(d). As can be seen from Figures 10(c) and $10(\mathrm{~d})$, the displacement response and velocity response of the structure decrease when the frequency ratio $\mu$ increases from 1.5 to 4 and then to 10 in the region where the structures collide. Compared with the case without pounding, pounding enlarges the displacement response of the right-side structure, so, with the increase of frequency ratio $\mu$, the enlargement effect of pounding on the right-side structure decreases gradually.

4.3. Effects of Structural Spacing $\Pi_{d}$. Figure 8 shows the response curves of dimensionless peak displacement $\Pi_{u}$ and dimensionless peak velocity $\Pi_{v}$ of adjacent inelastic SDOF structures corresponding to different dimensionless angular frequencies $\Pi_{\omega 1}$ of structures under different 


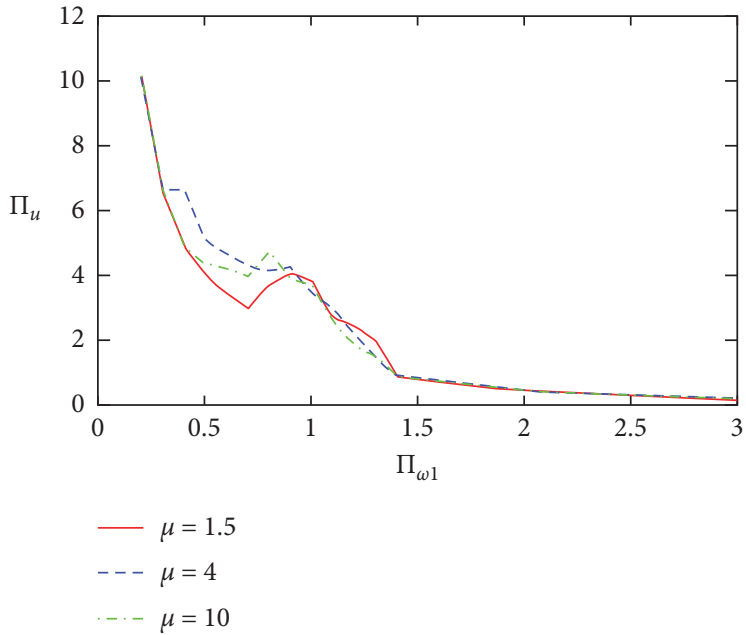

(a)

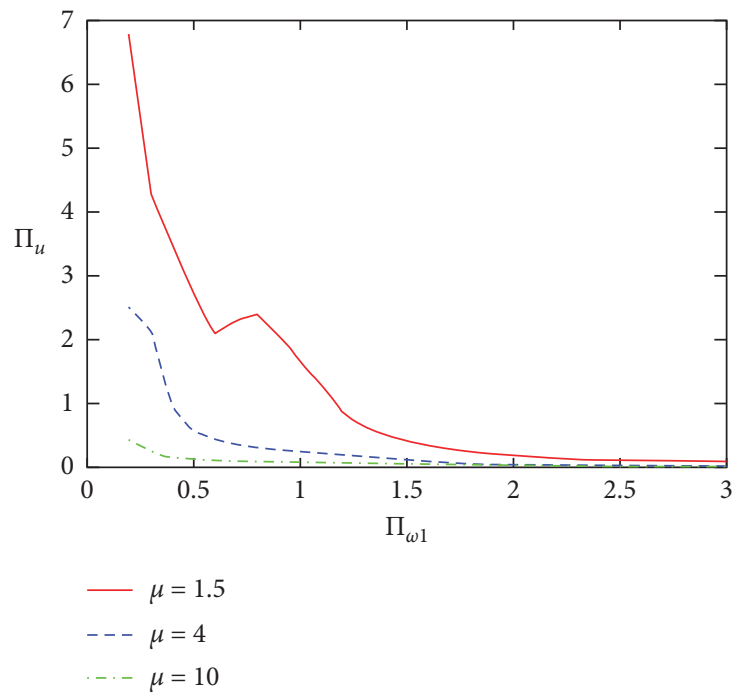

(c)

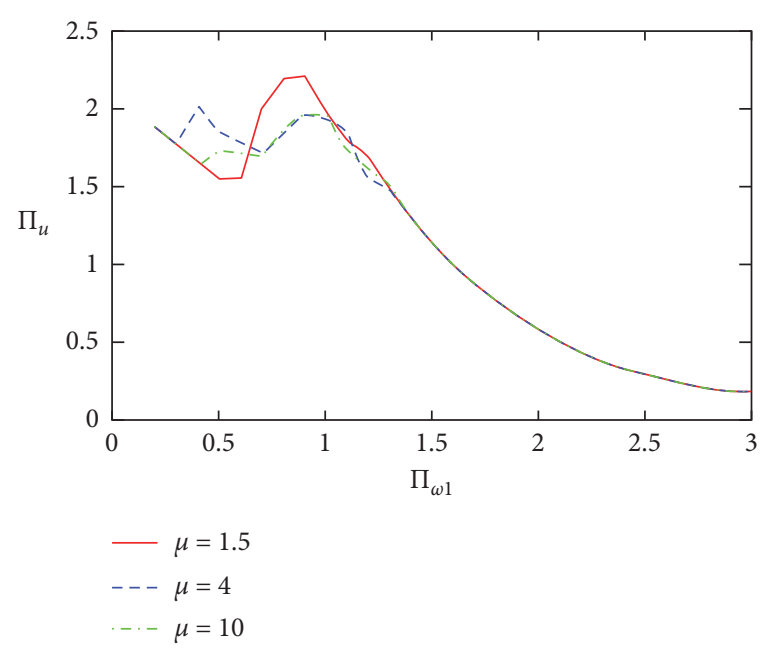

(b)

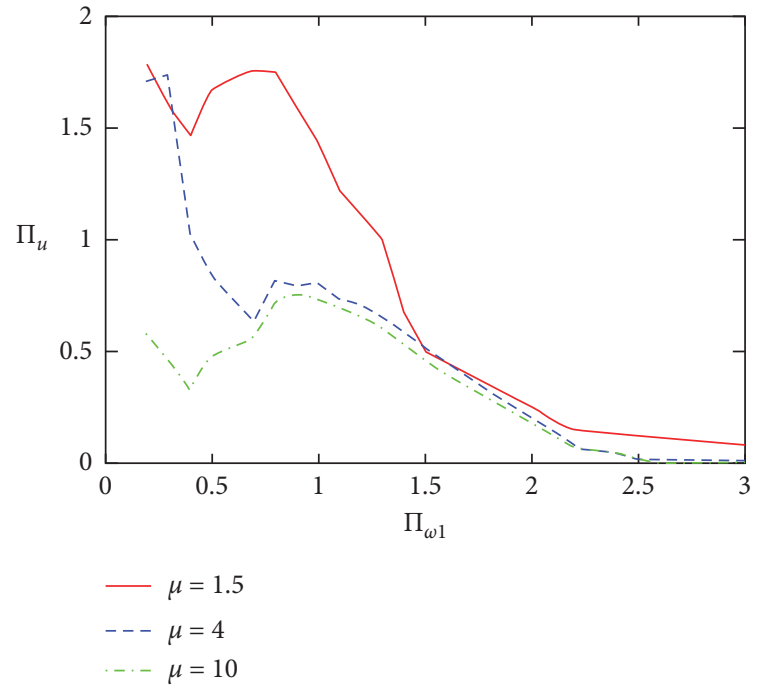

(d)

FIgURE 9: Maximum dimensionless displacement $\Pi_{u}$ and velocity $\Pi_{v}$ versus $\Pi_{\omega 1}$ for various mass ratios $\Pi_{m}$ $\left(\Pi_{\xi}=0.05, \Pi_{u y 1}=0.9 \Pi_{u y 2}=0.8, \Pi_{u y 1}=0.9, \mu=1.5, \Pi_{\alpha}=0.1, \Pi_{\omega c o n}=50, \Pi_{r}=0.4, \Pi_{d}=0.2\right)$. (a) Maximum dimensionless displacement $\Pi_{u}$ of left-side structure. (b) Maximum dimensionless velocity $\Pi_{v}$ of left-side structure. (c) Maximum dimensionless displacement $\Pi_{u}$ of right-side structure. (d) Maximum dimensionless velocity $\Pi_{v}$ of right-side structure.

structural spacing $\Pi_{d}$. Three structural spacing values are selected here, $\Pi_{d}=0.2, \Pi_{d}=1.5$, and the value of $\Pi_{d}$ is large enough so no pounding occurs between the two adjacent structures.

Figures $8(\mathrm{a})$ and $8(\mathrm{~b})$ show that when $0.7<\Pi_{\omega 1}<1.3$, the value of the curve corresponding to $\Pi_{d}=0.2$ is greater than that corresponding to $\Pi_{d}=1.5$. The curve corresponding to no pounding is the smallest. The results show that, with the increase of the spacing between the adjacent inelastic SDOF structures, the magnification of the impact on the left-side structure (with smaller mass and stiffness) decreases. When $1.3<\Pi_{\omega 1}<1.9$, the curves corresponding to $\Pi_{d}=1.5$ and no pounding coincide. It shows that the structural spacing is large when $\Pi_{d}=1.5$, and the impact of pounding between the two adjacent structures has little effect. In this section, the pounding will restrain the displacement response of the structure. At this time, the response of the structure for $\Pi_{d}=1.5$ is larger than that of the corresponding response for $\Pi_{d}=0.2$, so the response of the left-side structure will increase with the increase of the spacing of the structure. With the increase of $\Pi_{\omega 1}$, pounding has little effect on the left-side structure. In summary, the influence of structural spacing $\Pi_{d}$ on the left-side structure is also corresponding to the division of the three spectral regions. For the right-side structure (both the mass and stiffness are larger), as shown in Figures $8(\mathrm{c})$ and $8(\mathrm{~d})$, the displacement and velocity responses decrease with the increase of the spacing $\Pi_{d}$ of the structure, and the amplification of pounding decreases. 


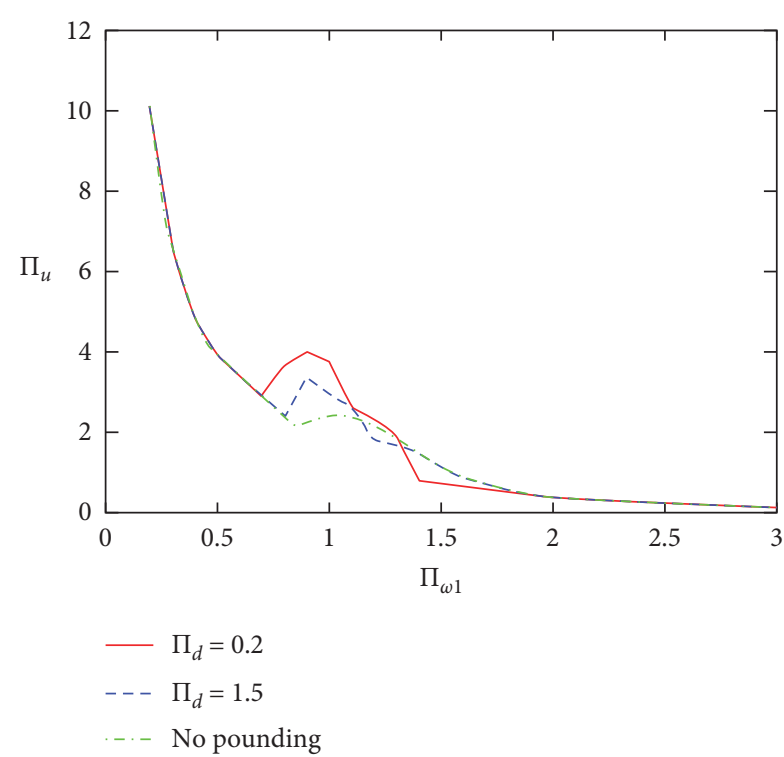

(a)

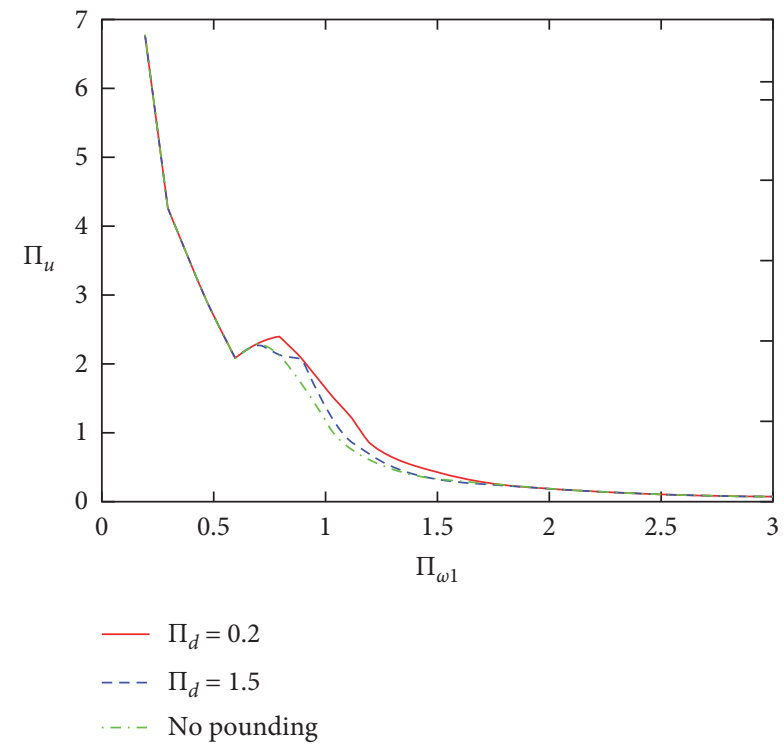

(c)

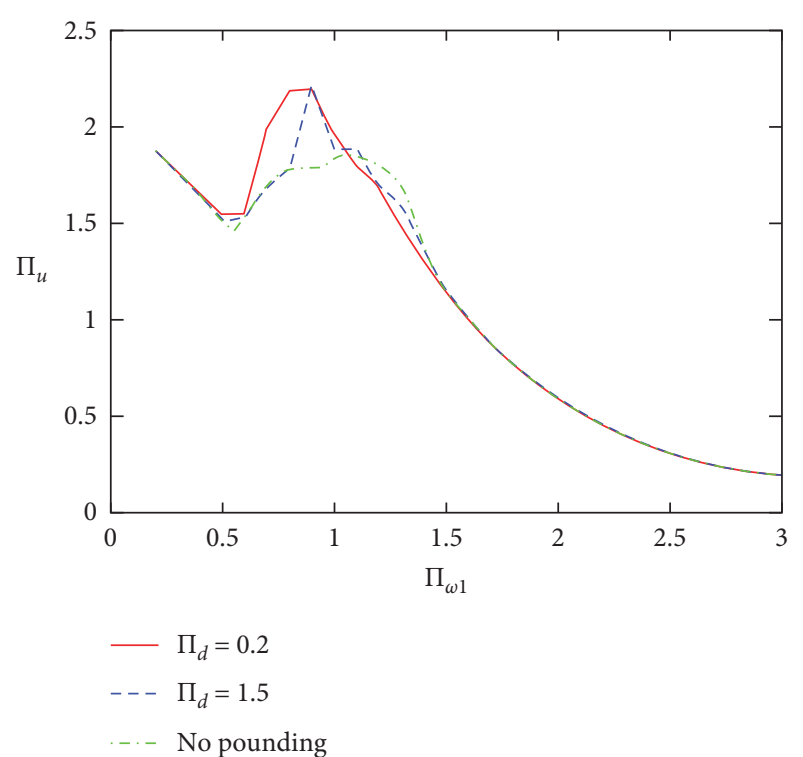

(b)

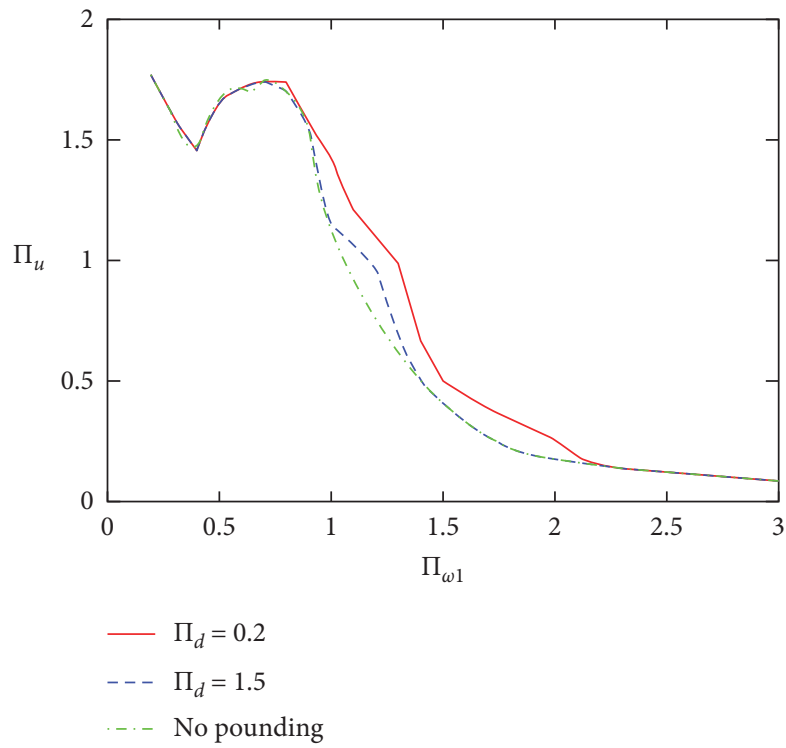

(d)

Figure 10: Maximum dimensionless displacement $\Pi_{u}$ and velocity $\Pi_{v}$ versus $\Pi_{\omega 1}$ for various frequency ratios $\mu\left(\Pi_{m}=0.5, \Pi_{\xi}=0.05\right.$, $\Pi_{u y 1}=0.9, \Pi_{u y 2}=0.8, \Pi_{u y 1}=0.9, \Pi_{\alpha}=0.1, \Pi_{\omega c o n}=50, \Pi_{r}=0.4$, and $\Pi_{d}=0.2$ ). (a) Maximum dimensionless displacement $\Pi_{u}$ of left-side structure. (b) Maximum dimensionless velocity $\Pi_{v}$ of left-side structure. (c) Maximum dimensionless displacement $\Pi_{u}$ of right-side structure. (d) Maximum dimensionless velocity $\Pi_{v}$ of right-side structure.

\section{Conclusions}

In this paper, the dimensional analysis method is used to study the pounding response of two inelastic SDOF structures under simplified earthquake excitation. The improved Kelvin pounding model is used to simulate the force and deformation of the collider during the contact process. The correctness and superiority of the improved Kelvin model are verified by comparing the pounding responses between the improved Kelvin model and Kelvin model. By studying the dimensional and dimensionless pounding responses of adjacent structures under different excitation acceleration amplitudes, the self-similarity of poundings between two inelastic SDOF structures is proved. The pounding response of two inelastic SDOF structures is studied in the form of spectra. The response of the pounding to the left-side structure (with smaller mass and stiffness) is divided into three spectral regions (amplification region, suppression region, and nonimpact region). However, the displacement and velocity responses of the right-side structure (with larger mass and stiffness) increase after pounding, and there are no three spectral regions.

The effects of structural parameters on the pounding response of two adjacent inelastic SDOF structures are also 
studied in this paper. The results show that the influence of structural parameters on the pounding response of structures with smaller mass and stiffness is closely related to the three spectral regions. For structures with larger mass and stiffness, the magnifying effect of pounding on structural response increases with the increase of mass ratio $\Pi_{m}$ and decreases with the increase of frequency ratio $\mu$ and structural spacing $\Pi_{d}$ between the adjacent inelastic SDOF structures.

(1) When the mass ratio $\Pi_{m}$ of the adjacent inelastic SDOF structures increases, the impact of pounding on the displacement and velocity response (including magnification and suppression) of the left-side structure (both mass and stiffness are smaller) decreases significantly. However, the displacement and velocity responses of the right-side structure (both mass and stiffness are larger) increase with the increase of the mass ratio $\Pi_{m}$.

(2) The displacement and velocity responses of the leftside structure increase first and then decrease with the increase of the frequency ratio $\mu$ of the adjacent inelastic SDOF structures in the first spectral region. In the second and third spectral regions, the effect of pounding on the displacement and velocity of the left-side structure is not obvious with the variety of frequency ratio $\mu$. Furthermore, the amplification effect of pounding on the displacement and velocity responses of the right-side structure decreases with the increase of frequency ratio $\mu$.

(3) As the structural spacing $\Pi_{d}$ increases, the amplification of pounding in the first spectral region decreases gradually for the left-side structure. In the second spectral region, the inhibition of pounding decreases gradually. The pounding effect in the third spectral region is not affected by the variety of structural spacing $\Pi_{d}$. However, the enlargement effect of pounding on the right-side structure decreases with the increasing of the spacing $\Pi_{d}$ between structures.

\section{Data Availability}

Some or all data, models, or code generated or used during the study are available from the corresponding author by request.

\section{Conflicts of Interest}

The authors declare that they have no conflicts of interest.

\section{Acknowledgments}

The authors are grateful to the National Natural Science Foundation of China (Grant no. 52078395). They also thank for the support by the Open Research Fund of State Key Laboratory of Geomechanics and Geotechnical Engineering, Institute of Rock and Soil Mechanics, Chinese Academy of Sciences (Grant no. Z018013), and Key Laboratory of
Earthquake Engineering and Engineering Vibration, Institute of Engineering Mechanics, China Earthquake Administration (Grant no. 2019D01).

\section{References}

[1] P. Zhu, M. Abe, and Y. Fujino, "Evaluation of pounding countermeasures and serviceability of elevated bridges during seismic excitation using 3D modeling," Earthquake Engineering \& Structural Dynamics, vol. 33, no. 5, pp. 591-609, 2004.

[2] C.-J. Wang and M.-H. Shih, "Performance study of a bridge involving sliding decks and pounded abutment during A violent earthquake," Engineering Structures, vol. 29, no. 5, pp. 802-812, 2007.

[3] C.-J. Wang, "Failure study of a bridge subjected to pounding and sliding under severe ground motions," International Journal of Impact Engineering, vol. 34, no. 2, pp. 216-231, 2007.

[4] F. D. Julian, T. Hayashikawa, and T. Obata, "Seismic performance of isolated curved viaducts equipped with deck unseating prevention cable restrainers," Journal of Constructional Steel Research, vol. 63, no. 2, pp. 237-253, 2007.

[5] National Research Council, The Great Alaska Earthquake of 1963, National Academy of Sciences, Washington, DC, USA, 1970.

[6] E. Rosenblueth and R. Meli, "The 1985 earthquake: causes and effects in Mexico city," Concrete International, vol. 8, no. 5, pp. 23-34, 1986.

[7] F. Naeim, M. Lew, S. C. Huang, H. K. Lam, and L. D. Carpenter, "The performance of tall buildings during the 21 september 1999 chi-chi earthquake, taiwan," The Structural Design of Tall Buildings, vol. 9, no. 2, pp. 137-160, 2000.

[8] Y. Ren, R. Wen, H. Yamanaka, and T. Kashima, "Site effects by generalized inversion technique using strong motion recordings of the 2008 Wenchuan earthquake," Earthquake Engineering and Engineering Vibration, vol. 12, no. 2, pp. 165-184, 2013.

[9] G. Sun, Y. G. Li, X. X. Du et al., "Earthquake disaster and emergency response overview in 2017," China Emergency Rescue, vol. 67, no. 1, pp. 9-14, 2018.

[10] J. P. Wolf and P. E. Skrikerud, "Mutual pounding of adjacent structures during earthquakes," Nuclear Engineering and Design, vol. 57, no. 2, pp. 253-275, 1980.

[11] K. T. Chau and X. X. Wei, "Pounding of structures modelled as non-linear impacts of two oscillators," Earthquake Engineering \& Structural Dynamics, vol. 30, no. 5, pp. 633-651, 2001.

[12] S. A. Anagnostopoulos, "Pounding of buildings in series during earthquakes," Earthquake Engineering \& Structural Dynamics, vol. 16, no. 3, pp. 443-456, 1988.

[13] R. Jankowsk, "Non-linear viscoelastic modelling of earthquake-induced structural pounding," Earthquake Engineering and Structural Dynamics, vol. 34, pp. 595-611, 2005.

[14] S. Y. Sabegh and N. J. Milani, "Pounding force response spectrum for near-field and far-field earthquakes," Scientia Iranica A, vol. 19, no. 5, pp. 1236-1250, 2012.

[15] R. J. Zhang, Q. N. Li, and J. H. Yin, "Precise integration method of contact element model in seismic impact analysis," Vibration and Shock, vol. 35, no. 3, pp. 121-128, 2016.

[16] C. H. Zhai, S. Jiang, and Z. Q. Chen, "Dimensional analysis of the pounding response of an oscillator considering contact duration," Journal of Engineering Mechanics(ASCE), vol. 141, no. 4, Article ID 04014138, 2015. 
[17] Q. M. Tan, Dimensional Analysis, Press of University of Science and Technology of China, London, UK, 2007.

[18] N. Makris and C. J. Black, "Dimensional analysis of rigidplastic and elastoplastic structures under pulse-type excitations," Journal of Engineering Mechanics, vol. 130, no. 9, pp. 1006-1018, 2004.

[19] N. Makris and C. J. Black, "Dimensional analysis of bilinear oscillators under pulse-type excitations," Journal of Engineering Mechanics, vol. 130, no. 9, pp. 1019-1031, 2004.

[20] J. Zhang and Y. Tang, "Dimensional analysis of structures with translating and rocking foundations under near-fault ground motions," Soil Dynamics and Earthquake Engineering, vol. 29, no. 10, pp. 1330-1346, 2009.

[21] E. Dimitrakopoulos, N. Makris, and A. J. Kappos, "Dimensional analysis of the earthquake-induced pounding between adjacent structures," Earthquake Engineering \& Structural Dynamics, vol. 38, no. 7, pp. 867-886, 2009.

[22] E. Dimitrakopoulos, A. J. Kappos, and N. Makris, "Dimensional analysis of yielding and pounding structures for records without distinct pulses," Soil Dynamics and Earthquake Engineering, vol. 29, no. 7, pp. 1170-1180, 2009.

[23] E. Dimitrakopoulos, N. Makris, and A. J. Kappos, "Dimensional analysis of the earthquake response of a pounding oscillator," Journal of Engineering Mechanics, vol. 136, no. 3, pp. 299-310, 2010.

[24] Q. Y. Wu, T. Wang, H. B. Ge, and H. P. Zhu, "Dimensional analysis of pounding response of an oscillator based on modified Kelvin pounding model[J]," Journal of Aerospace Engineering(ASCE), vol. 32, no. 4, Article ID 04019039, 2019.

[25] K. Ye, L. Li, and H. Zhu, "A modified Kelvin impact model for pounding simulation of base-isolated building with adjacent structures," Earthquake Engineering and Engineering Vibration, vol. 8, no. 3, pp. 433-446, 2009.

[26] J. Penizen, "Dynamic response of elasto-plastic frames," Journal of Structural Division(ASCE), vol. 127, no. 2, pp. 1-13, 1962.

[27] S. Jiang, Dimensional Analysis of Earthquake-Induced Pounding between Adjacent Buildings, Harbin Institute of Technology, London, UK, 2015.

[28] N. M. Newmark, "A method of computation for structural dynamics," Journal of the Engineering Mechanics Division, vol. 85, no. 3, pp. 67-94, 1959. 